\title{
MAGNETIC RESONANCE IMAGING OF LOW-GRADE FIBROMYXOID SARCOMA
}

Martin Torriani, ${ }^{\mathrm{a}}$ Mauricio Etchebehere, ${ }^{\mathrm{b}}$ Eliane M. I. Amstalden, ${ }^{\mathrm{c}}$ and Hugue Ouellette ${ }^{\mathrm{a}}$

Low-grade fibromyxoid sarcoma (LGFMS) is a rare soft-tissue tumor with a deceptively benign histologic appearance affecting predominantly young adults during the fourth decade of life ${ }^{1}$. Low-grade fibromyxoid sarcoma has a predilection for involving deep soft tissues of the thigh, inguinal region, or chest wall, affecting less frequently the shoulder or axilla ${ }^{1}$. Local postsurgical recurrence and metastases to lungs and bone are frequently seen ${ }^{1}$. The surgical management, histopathologic findings, and biological behavior of LGFMS have been outlined in the literature $^{1,2}$. There are very few reports on the magnetic resonance (MR) imaging features of LGFMS ${ }^{3,4}$. We present the MR imaging features of a surgically confirmed case of LGFMS affecting the shoulder.

A 30-year-old man presented with a 20-year history of a painless slow-growing mass in the right shoulder. Magnetic resonance images were obtained on a 2.0T scanner (Elscint, Haifa, Israel), demonstrating a well-defined softtissue mass measuring $12.0 \times 7.0 \times 9.0 \mathrm{~cm}$ located between the deltoid muscle, rotator-cuff muscles, and proximal humerus. No peri-tumoral edema in adjacent subcutaneous tissues or muscles was noted. The signal intensity (SI) of the bone marrow was normal. The mass had an intermediate, heterogeneous SI on T1-weighted images [repetition time (TR) / time to echo (TE), 600/25] (Fig. 1a). Heterogeneous low to high SI with multiple hypointense intralesional nodules was seen on T2-weighted fast spin-echo images (TR/TE, 3500/90) (Fig. 1b). The mass enhanced heterogeneously on T1-weighted fat-suppressed images (TR/TE, 720/20) after intravenous injection of gadolinium (Fig. 1c), predominantly in corresponding $\mathrm{T} 2$-weighted hyperintense areas.

aDivision of Musculoskeletal Radiology, Department of Radiology, Massachusetts General Hospital and Harvard Medical School - Boston, MA, USA.

'Department of Orthopaedics, Medical Sciences School, State University of Campinas - Campinas/SP, Brazil.

'Department of Pathology, Medical Sciences School, State University of Campinas - Campinas/SP, Brazil.

Email: mtorriani@hms.harvard.edu
The lesion was surgically removed with negative margins. The surgical specimen consisted of a soft-tissue tumor, measuring $14.5 \times 9.5 \times 5.0 \mathrm{~cm}$ and weighing $290 \mathrm{~g}$. The cut sections showed a firm, grossly circumscribed, and lobulated mass, with a yellow-white color and glistening appearance secondary to the accumulation of myxoid ground substance (Fig. 2a). Microscopically, the tumor had low to moderate cellularity composed of bland spindleshaped cells with a fibroblast pattern, arranged mainly in

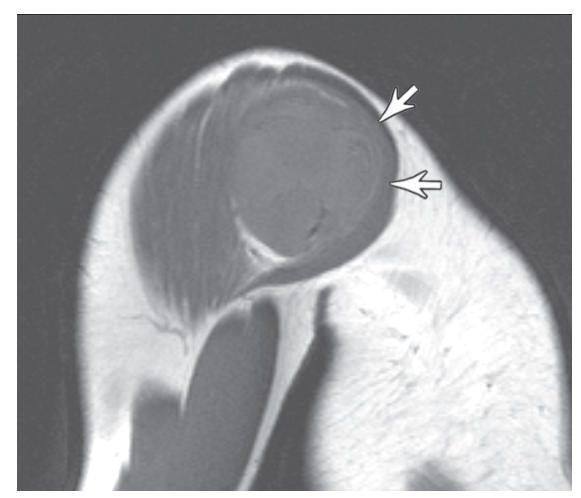

Figure 1a - Sagittal T1-weighted MR image of the shoulder shows a welldefined mildly heterogeneous soft tissue mass of intermediate signal intensity displacing the deltoid muscle (arrows)

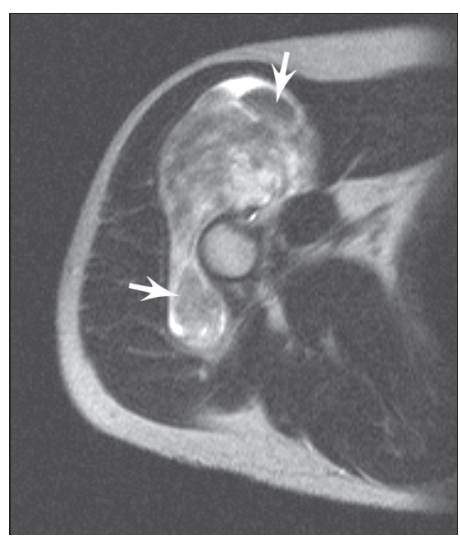

Figure 1b-Axial T2-weighted fast spin-echo MR image shows well-defined mass between deltoid and proximal humerus of heterogeneous signal intensity. Nodular high and low signal areas are seen (arrows) 


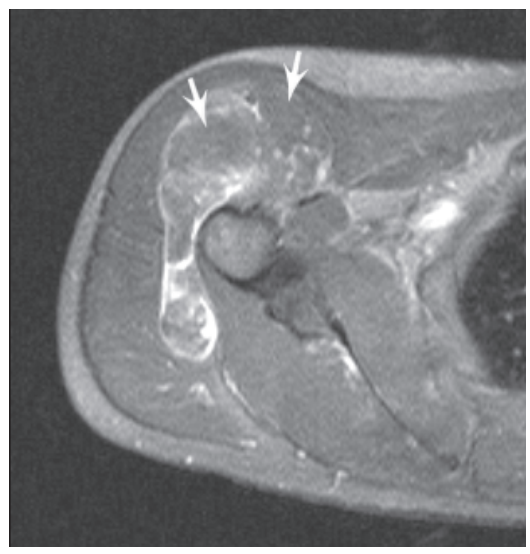

Figure 1c - Axial fat-suppressed T1-weighted MR image after intravenous injection of gadolinium-DTPA shows heterogeneous enhancement, predominantly in corresponding to T2-hyperintense (likely myxoid) areas (arrows)

a whorled fashion and amidst a variable fibrous and myxoid stroma. The myxoid zones alternated abruptly with the fibrous zones (Fig. 2b). The cells had indistinct pale eosinophilic cytoplasm and showed mild nuclear pleomorphism with low mitotic activity. Immunohistochemically, the neoplastic cells stained strongly and diffusely for vimentin and did not express immunoreactivity to muscle-specific actin, cytokeratins, S-100 protein, or CD34. A histopathological diagnosis of LGFMS was made. The patient had

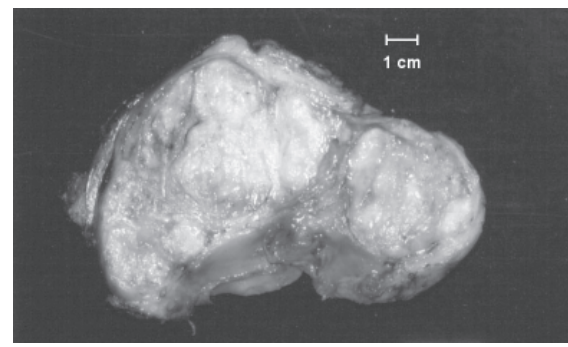

Figure 2a - Macroscopic features. A grossly circumscribed and lobulated yellow-white mass, with a glistening surface due to myxoid ground substance

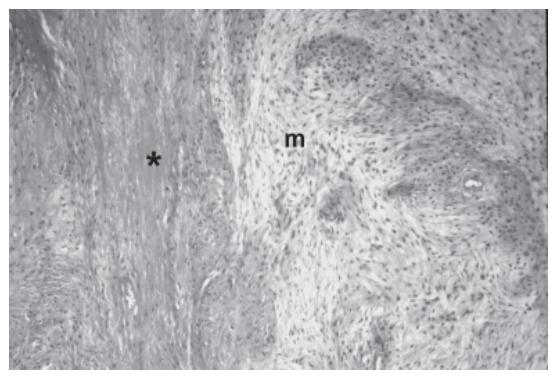

Figure 2b - Microscopic features. Alternating areas of fibrous (asterisk) and myxoid (m) stroma in a whorled growth pattern (hematoxylin and eosin, 63X). These findings associated with diffuse staining for vimentin (not shown) characterize low-grade fibromyxoid sarcoma no evidence of local recurrence or metastases after 2 years of follow-up.

Low-grade fibromyxoid sarcoma was first recognized by Evans in 1987, when he described a bland fibromyxoid neoplasm arising in the deep soft tissues of 2 young women with subsequent development of metastases ${ }^{5}$. Although this is a rare entity, it is reasonable to speculate that it is probably more common than reported in the medical literature due to the likelihood of misdiagnosis with other low-grade fibrous or myxoid soft-tissue neoplasms.

Low-grade fibromyxoid sarcoma occurs more frequently in young to middle-aged adults; however, cases have been reported in patients between 6 to 65 years of age. Males are affected more commonly than females. ${ }^{1,6}$ The usual presentation is a slow-growing painless mass. Low-grade fibromyxoid sarcoma is frequently surrounded by skeletal muscle $^{1,6}$ and has a predilection for involving deep soft tissues of the thigh, inguinal region, or chest wall. Less frequently, it can involve the shoulder or axilla. ${ }^{7}$ Low-grade fibromyxoid sarcoma frequently recurs and can metastasize, especially to the lungs, ${ }^{1}$ with patients surviving between 10 and 15 years after detection of metastases ${ }^{1,6}$. Due to the rarity of this neoplasm, the role of chemotherapy and radiotherapy remains unknown.

The gross findings of LGFMS include a well-circumscribed mass with a mean tumor size at diagnosis of 9.5 $\mathrm{cm} .{ }^{1}$ On cut section, LGFMS has a yellow-white or greywhite appearance with focal areas of gelatinous myxoid tissue, rarely exhibiting hemorrhage or necrosis. Histologically, although a pseudocapsule surrounds the tumor, focal microscopic infiltration into the adjacent soft tissues has been reported. ${ }^{8}$ Histologically, LGFMS is of low or moderate cellularity, with swirling fibrous and myxoid areas with deceptively benign-appearing fibroblastic spindle cells. Mitotic figures are uncommon, and nuclear pleomorphism is usually absent or rare. ${ }^{1}$ Immunohistochemically, the tumor cells stain strongly and diffusely for vimentin, and smooth muscle actin or muscle-specific actin may be present. ${ }^{69}$ Supernumerary ring chromosomes are a hallmark of LGFMS. ${ }^{10}$

A prior report on the imaging characteristics of LGFMS described a heterogeneously hyperechogenic multi-nodular sonographic appearance, a heterogeneous MR imaging appearance with low to slightly high SI on T1-weighted images, heterogeneously low to high SI on T2-weighted images, and heterogeneous postcontrast enhancement. ${ }^{3}$ In our case, MR imaging showed a well-defined mass with heterogeneous intermediate to low SI on T1-weighted images, and heterogeneous high SI on T2-weighted images with hypointense nodular areas. Postcontrast T1-weighted images showed heterogeneous enhancement mostly in the 
periphery of the mass. Macroscopic analysis of cut specimens compared to MR imaging findings suggested that focal areas of low T2 SI may correlate with the fibrous areas of the tumor. In comparison with the 2 cases described by Koh et al, ${ }^{3}$ our findings are comparable to their case describing LGFMS affecting the chest wall. In our case, we did not observe ring-like structures on MR imaging or macroscopic examination. This underscores the variability that may be present in the several components that constitute LGFMS.

The differential diagnosis of LGFMS includes several benign and malignant neoplasms containing variable amounts of myxoid and fibrous tissue. Neurofibromas and malignant peripheral nerve sheath tumors may present his- tological and imaging findings similar to LGFMS. ${ }^{3}$ The myxoid zones of LGFMS may resemble myxoid liposarcoma. ${ }^{11}$ Histologically, the most important differential diagnosis is with myxofibrosarcoma, which commonly arises in the subcutaneous tissues of elderly patients and is uniformly myxoid without alternating fibrous zones. Such a distinction is important, as myxofibrosarcoma rarely metastasizes, in contrast to LGFMS. ${ }^{11}$

Low-grade fibromyxoid sarcoma is a rare soft tissue tumor with slow growth and deceptively benign histologic appearance. The possibility of LGFMS must be considered when elaborating differential diagnostic possibilities for young adults with a large soft tissue mass exhibiting MR imaging characteristics of intermixed fibrous and myxoid tissue.

\section{REFERENCES}

1. Evans HL. Low-grade fibromyxoid sarcoma. A report of 12 cases. Am J Surg Pathol. 1993;17:595-600.

2. van den Bossche MR, Van Mieghem H. Low-grade fibromyxoid sarcoma. Oncology. 2000;58:207-9.

3. Koh SH, Choe HS, Lee IJ, Park HR, Bae SH. Low-grade fibromyxoid sarcoma: ultrasound and magnetic resonance findings in two cases. Skeletal Radiol. 2004.

4. De Schepper AM, De Beuckeleer L, Vandevenne J, Somville J. Magnetic resonance imaging of soft tissue tumors. Eur Radiol. 2000;10:213-23.
5. Evans HL. Low-grade fibromyxoid sarcoma. A report of two metastasizing neoplasms having a deceptively benign appearance. Am J Clin Pathol. 1987;88:615-9.

6. Goodlad JR, Mentzel T, Fletcher CD. Low grade fibromyxoid sarcoma: clinicopathological analysis of eleven new cases in support of a distinct entity. Histopathology. 1995;26:229-37.

7. Shidham VB, Ayala GE, Lahaniatis JE, Garcia FU. Low-grade fibromyxoid sarcoma: clinicopathologic case report with review of the literature. Am J Clin Oncol. 1999;22:150-5. 
8. Lindberg GM, Maitra A, Gokaslan ST, Saboorian MH, Albores-Saavedra J. Low grade fibromyxoid sarcoma: fine-needle aspiration cytology with histologic, cytogenetic, immunohistochemical, and ultrastructural correlation. Cancer. 1999;87:75-82.

9. Canpolat C, Evans HL, Corpron C, Andrassy RJ, Chan K, Eifel P, et al. Fibromyxoid sarcoma in a four-year-old child: case report and review of the literature. Med Pediatr Oncol. 1996;27:561-4.
10. Mezzelani A, Sozzi G, Nessling M, Riva C, Della Torre G, Testi MA, et al. Low grade fibromyxoid sarcoma. a further low-grade soft tissue malignancy characterized by a ring chromosome. Cancer Genet Cytogenet. 2000;122:144-8.

11. Enzinger F, Weiss, SW. Soft Tissue Tumors. 2001;4th edition p. 42531. 